\title{
Reductant-Activated, High-Coverage, Covalent Functionalization of ${ }^{1} \mathrm{~T}^{\prime}-\mathrm{MoS}_{2}$ Supporting Information
}

Ellen X. Yan, Miguel Cabán-Acevedo, Kimberly M. Papadantonakis, Bruce S. Brunschwig, and Nathan S. Lewis

Division of Chemistry and Chemical Engineering, California Institute of Technology, Pasadena, California 91125, United States

\section{Table of Contents}

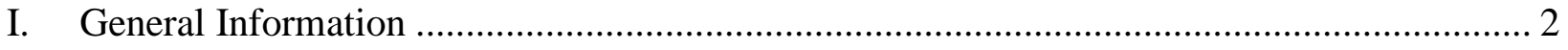

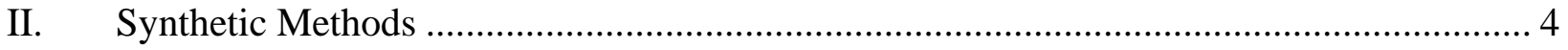

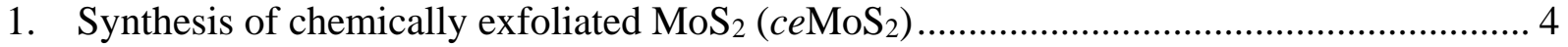

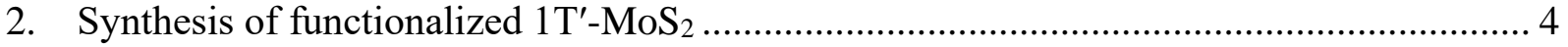

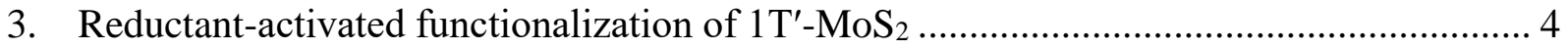

III. Calculations....................................................................................................... 4

1. Analysis and quantification of coverage from XPS data ……............................................ 4

2. Quantification of coverage from ${ }^{13} \mathrm{C}$ MAS NMR data ....................................................... 5

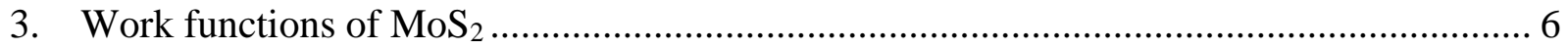

4. Effective reduction potential for one-electron reductants................................................... 6

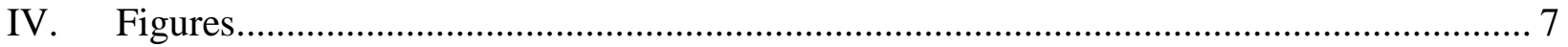

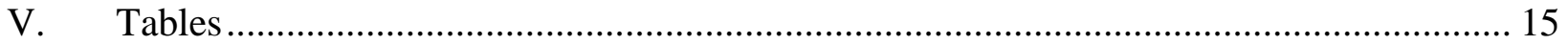

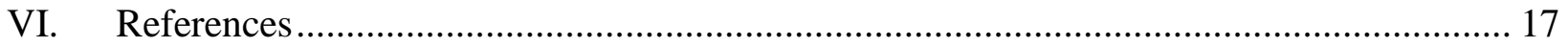




\section{General Information}

All organic solvents and reagents were purchased commercially and used as received without further purification. Molybdenum disulfide powder (99\%), bis(cyclopentadienyl)nickel(II) (nickelocene), and bis(tetramethylcyclopentadienyl)nickel(II) (octamethylnickelocene) were purchased from Alfa Aesar. Anhydrous organic solvents, $n$-butyllithium (1.6 M in hexanes), and bis(cyclopentadienyl)cobalt(II) (cobaltocene) were purchased from Sigma-Aldrich. All of the above chemicals were stored in an Ar-filled glovebox $\left(<1 \mathrm{ppm} \mathrm{O}_{2}\right)$. Nanopure water with a resistivity of $\geq 18.2 \mathrm{M} \Omega \cdot \mathrm{cm}$ was obtained from a Barnstead E-Pure system. The centrifuge used for purification was a Beckman Coulter Avanti J-20 XP centrifuge with a JA-17 fixed rotor, where $\operatorname{rcf}(\times \mathrm{g})=137.75 \times(\mathrm{rpm} / 1000)^{2}$.

X-ray photoelectron spectroscopy (XPS) and ultraviolet photoelectron spectroscopy (UPS) data were collected using a Kratos AXIS Ultra spectrometer equipped with a hybrid magnetic and electrostatic electron lens system and a delay-line detector (DLD). The photoelectron-ejection vector was $90^{\circ}$ with respect to the sample surface plane. XPS data were collected using a monochromatic $\mathrm{Al} \mathrm{K \alpha} \mathrm{X}$-ray source $(1486.7 \mathrm{eV})$ at pressures $<9 \times 10^{-9}$ Torr. The electroncollection lens aperture was set to sample a $700 \times 300 \mu \mathrm{m}$ spot, and the analyzer pass energy was $80 \mathrm{eV}$ for survey spectra and $10 \mathrm{eV}$ for high-resolution spectra. UPS data were collected using a He I ultraviolet source $(21.2 \mathrm{eV})$ at a pressure of $1 \times 10^{-8}$ Torr in the analysis chamber. The electron-collection lens aperture was set to a $55 \mu \mathrm{m}$ spot size and the analyzer pass energy was 5 $\mathrm{eV}$. The instrument energy scale and work function were calibrated using clean $\mathrm{Au}, \mathrm{Ag}$, and $\mathrm{Cu}$ standards. The instrument was operated by Vision Manager software v. 2.2.10 revision 5 and data were analyzed using CasaXPS software (CASA Software Ltd).

Attenuated total reflectance Fourier transform infrared spectroscopy (ATR-FTIR) data were collected using a Thermo Scientific Nicolet 6700 optical spectrometer with a IR6118 Diamond ATR Smart iTR accessory. The spectrometer was equipped with a deuterated L-alanine-doped triglycine sulfate (DLaTGS) detector, an electronically temperature-controlled (ETC) EverGlo mid-IR source, a $\mathrm{N}_{2}(\mathrm{~g})$ purge, and a $\mathrm{KBr}$ beam splitter. The spectra reported herein are averages of 1000 scans at $2 \mathrm{~cm}^{-1}$ resolution, background corrected using the spectrum of $c e \mathrm{MoS}_{2}$. Spectra were collected and processed using OMNIC software v. 9.2.41.

Raman spectra were collected with a Renishaw inVia Raman microprobe equipped with a Leica DM 2500 M microscope, a Leica N Plan 50x objective (numerical aperture $=0.75$ ), a 1800 lines $\mathrm{mm}^{-1}$ grating, and a CCD detector configured in a $180^{\circ}$ backscatter geometry. A $532 \mathrm{~nm}$ diodepumped solid-state (DPSS) laser (Renishaw RL532C50) was used as the excitation source. A $\lambda / 4$ plate was used to circularly polarize the incident excitation. No polarizing collection optic was used.

The solid-state carbon-13 (125.4 MHz) nuclear magnetic resonance (NMR) spectra were acquired on a Bruker DSX-500 MHz NMR spectrometer using a Bruker $4.0 \mathrm{~mm}$ magic-angle spinning probe at ambient conditions. Samples were prepared by carefully packing dried functionalized $\mathrm{MoS}_{2}$ powder in a zirconium rotor. For ${ }^{13} \mathrm{C}$ cross-polarization magic angle spinning $\left({ }^{13} \mathrm{C} C P M A S\right)$ NMR experiments, $8.0 \mathrm{kHz}$ spin rate, $4 \mathrm{~s}$ recycle delay, $2 \mathrm{~ms}$ contact time, $\pi / 2$ pulse width of $4 \mu \mathrm{s}$, and typically 4000 scans using a TPPI ${ }^{1} \mathrm{H}$ decoupling method were used. The spectra were referenced to tetramethylsilane (TMS). 
A Cahn C-35 microbalance having a sensitivity of $10 \mu \mathrm{g}$ was used to determine the concentration of $\mathrm{MoS}_{2}$ dispersions. 


\section{Synthetic Methods}

\section{Synthesis of chemically exfoliated $\operatorname{MoS}_{2}\left(c e \mathrm{MoS}_{2}\right)$}

To intercalate $\mathrm{Li}, \mathrm{MoS}_{2}$ powder (400 mg) was heated with $n$-butyllithium (1.6 M in hexanes, $4 \mathrm{~mL}$ ) for 46 hours in a sealed glass tube. The powder was filtered and washed with anhydrous hexanes $(10 \times 2 \mathrm{~mL})$, then exfoliated in $180 \mathrm{~mL}$ of $\mathrm{H}_{2} \mathrm{O}$. The dispersion was sonicated for $1 \mathrm{~h}$, then centrifuged at $2000 \mathrm{rpm}(551 \mathrm{rcf}(\mathrm{xg}))$ for $5 \mathrm{~min}$ to remove unexfoliated material. The supernatant was retained and washed twice with $\mathrm{H}_{2} \mathrm{O}$, and optionally washed with anhydrous DMF $(\mathrm{N}, \mathrm{N}-$ dimethylformamide) for reactions conducted in DMF. The final precipitate was resuspended in either 2:1 water/isopropanol or DMF, depending on the reaction. Samples of $c e \mathrm{MoS}_{2}$ were dropcast from 1:1 water/methanol onto $p^{+}$-Si for XPS measurements, and were dried in vacuo for 2 days for ATR-FTIR and NMR measurements.

\section{Synthesis of functionalized $1 \mathbf{T}^{\prime}-\mathrm{MoS}_{2}$}

Exfoliated $\mathrm{MoS}_{2}$ was suspended in either 2:1 water/isopropanol or DMF at a concentration of 2 $\mathrm{mg} / \mathrm{mL}$ in a $20 \mathrm{~mL}$ scintillation vial (typically $10 \mathrm{~mL}$ ). The concentration was determined by evaporating $0.1 \mathrm{~mL}$ of solution and measuring the mass using a microbalance. All of the functionalized $\mathrm{MoS}_{2}$ (fct-MoS ) samples were synthesized using DMF as the solvent unless otherwise indicated. The alkyl halide was added (10 eq.) and the solution was stirred for $42 \mathrm{~h}$ while being covered in aluminum foil. Centrifugation to wash the fct-MoS $\mathrm{S}_{2}$ was performed at $6000 \mathrm{rpm}$ (4959 $\mathrm{rcf}(\times \mathrm{g})$ ) for $30 \mathrm{~min}$ rounds, and the precipitate was resuspended by sonication. in between rounds. The product was washed sequentially with isopropanol $(3 \times 12 \mathrm{~mL})$, methanol $(2 \times 12$ $\mathrm{mL})$, and water $(1 \times 12 \mathrm{~mL})$. The final product was resuspended in 1:1 water/methanol for dropcasting (XPS) and the solvent was removed in vacuo to obtain a dry powder.

\section{Reductant-activated functionalization of $1 \mathrm{~T}^{\prime}-\mathrm{MoS}_{2}$}

Functionalized $\mathrm{MoS}_{2}$ was suspended in DMF (10 mL) and the alkyl halide was added (10 eq.). Metallocenes were then added in either ambient conditions (ferrocene) or in an Ar-purged glovebox (nickelocene, octamethylnickelocene, cobaltocene) after purging the $\mathrm{MoS}_{2} / \mathrm{DMF}$ solution with $\operatorname{Ar}(\mathrm{g})$. The solution was covered with aluminum foil and stirred for $66 \mathrm{~h}$, then purified by centrifugation at $6500 \mathrm{rpm}(5820 \mathrm{rcf}(\times \mathrm{g}))$ in $10 \mathrm{~min}$ rounds, resuspending the precipitate by sonication in between rounds. The product was washed with DMF until the metallocene color was not visible (typically 3-4 rounds $\times 12 \mathrm{~mL})$, then with isopropanol $(2 \times 12 \mathrm{~mL})$, and methanol $(2 \times$ $12 \mathrm{~mL}$ ). The final product was resuspended in 1:1 water/methanol for dropcasting (XPS) and the solvent was removed in vacuo to obtain a dry powder.

\section{Calculations}

\section{Analysis and quantification of coverage from XPS data}

High-resolution XPS data were analyzed using CasaXPS software v. 2.3.17. A Shirley background was applied to all $\mathrm{C} 1 s, \mathrm{~S} 2 p$, and Mo $3 d$ spectra, with endpoints averaged from 21 points. Spectra were calibrated by setting the $\mathrm{C} 1 s$ peak at $284.8 \mathrm{eV}$. Peaks were fitted according to the constraints listed below, and the coverage per sulfur atom was obtained using the area of the functionalized sulfur peaks divided by the total area of all sulfur peaks. This value was doubled to obtain the 
coverage per $\mathrm{MoS}_{2}$ unit. Although a similar value can be obtained by quantifying the sulfur peaks relative to the Mo $3 d$ peaks, a comparison within the same XPS binding energy region removes errors associated with any changes in the inelastic mean free path (IMFP) of photoelectrons as the kinetic energy is changed.

\section{Lineshapes}

The Mo $3 d$ peaks were fit using a modified Lorentzian asymmetric lineshape with tail damping, $\operatorname{LF}(\alpha, \beta, w, m)$, where $\alpha$ and $\beta$ determine the tail asymmetry, $w$ is the tail damping parameter, and $m$ defines the width of the Gaussian. $\operatorname{LF}(1,1,55,260)$ was used to fit all Mo $3 \mathrm{~d}$ peaks based on previous reports modeling Mo XPS peaks. ${ }^{1}$ The $\mathrm{S} 2 s$ peak was fit using a Lorentzian asymmetric lineshape, $\operatorname{LA}(\alpha, \beta, m)$, with parameters $\operatorname{LA}(2,2,50)$ by matching the experimental data and minimizing the residual standard deviation (residual STD) between the fit and the data in CasaXPS. S $2 p$ peaks were fit using a Voigt GL(30) function with $70 \%$ Gaussian and $30 \%$ Lorentzian character, which resulted in a minimum in the residual STD.

\section{Constraints}

Due to spin-orbit coupling, the Mo $3 d$ and S $2 p$ peaks are doublets with specific area ratios and position constraints. The following constraints were placed on the Mo $3 d_{3 / 2}$ peak relative to the Mo3 $d_{5 / 2}$ peak: $\operatorname{area}\left(3 d_{5 / 2}\right) \times 0.67, \operatorname{FWHM}\left(3 d_{5 / 2}\right) \pm 0.2$, and position $\left(3 d_{5 / 2}\right)+3.13 \mathrm{eV}$. The S $2 p_{1 / 2}$ peaks were fit with the following constraints: area $\left(2 p_{1 / 2}\right)=\operatorname{area}\left(2 p_{3 / 2}\right) \times 0.5$, FWHM equal to that of the $2 p_{3 / 2}$ peak, and position $\left(2 p_{3 / 2}\right)+1.18 \mathrm{eV}$. In addition, the ratios of the areas and positions of the $2 \mathrm{H}$ - to $1 \mathrm{~T}^{\prime}-\mathrm{MoS}_{2} \mathrm{~S} 2 p$ peaks was determined from 4 samples of $c e \mathrm{MoS}_{2}$ and used to constrain the ratios of $2 \mathrm{H}$ to $1 \mathrm{~T}^{\prime}-\mathrm{MoS}_{2}$ in the analysis of functionalized samples to minimize over- or underfitting the functionalized sulfur peak. Thus, the $2 \mathrm{H}-\mathrm{MoS}_{2} \mathrm{~S} 2 p$ peak areas were constrained to be $18 \%$ of the total S $2 p$ area in fct-MoS 2 spectra (similar ratio constraint for the Mo $3 d$ spectra), and the peak position equal to $1 \mathrm{~T}^{\prime}-\mathrm{MoS}_{2}+1.32 \mathrm{eV}$. By the same reasoning for consistency, the $\mathrm{S}^{*}$ peak was constrained to be $1 \mathrm{~T}^{\prime}-\mathrm{MoS}_{2}-0.7 \mathrm{eV}$.

\section{Error Analysis}

A residual STD close to $1(\sim 0.97)$ was observed in the noise of the background in spectra, therefore Poisson statistics can be applied. Since we can assume Poisson distribution for the noise, Monte Carlo simulation can be used to estimate the errors in the fitting and test the reproducibility of the fitting given the constraints above. We applied Monte Carlo simulations using $n=400$ for number of simulations for all the data used in Figure 4 and found that the mean of the percent standard deviation (\%std) of the peak areas was within $2 \%$ for all cases except those of chloropropane where functionalization cannot be detected, resulting in an expected larger percentage error (Figure S13). Therefore, our fitting parameters are relatively robust.

\section{Quantification of coverage from ${ }^{13} \mathrm{C}$ MAS NMR data}

For quantification to corroborate the XPS analysis, solid state magic angle spinning (MAS) ${ }^{13} \mathrm{C}$ NMR spectra was obtained for ${ }^{13} \mathrm{C}$-enriched methyl-MoS 2 synthesized from ${ }^{13} \mathrm{C}$-iodomethane under three conditions: no reductant, with nickelocene, and with cobaltocene. Spectra were normalized using an external standard, 1,2,4,5-tetramethyl-1H-imidazole, and corrected for the natural abundance of ${ }^{13} \mathrm{C}$. The $\mathrm{mmol} / \mathrm{g}$ of carbon was calculated per sample according to the 
standard spectrum, and converted to coverage per $\mathrm{MoS}_{2}$ using the expected molecular weight of the functionalized samples based on the XPS data (i.e. for no reductant, with $\sim 40 \%$ coverage per $\mathrm{MoS}_{2}$, the molecular weight of $\mathrm{MoS}_{2}\left({ }^{13} \mathrm{CH}_{3}\right)_{0.4}$ was used). For example, with $2.4 \mathrm{mmol} / \mathrm{g}$ of carbon in the "no reductant" condition and $10.1 \mathrm{mg}$ of sample during the measurement:

$$
\text { coverage per } \begin{aligned}
\mathrm{MoS}_{2} & =(\mathrm{mmol} \text { of carbon }) /(\mathrm{mmol} \text { of Mo }) \\
& =(2.4 \mathrm{mmol} / \mathrm{g} \times 0.0101 \mathrm{~g}) /(0.0101 \mathrm{~g} /(166.5 \mathrm{~g} / \mathrm{mol}) \times 1000 \mathrm{mg} / \mathrm{g}) \\
& =0.02424 \mathrm{mmol} \mathrm{C} / 0.06066 \mathrm{mmol} \mathrm{Mo} \\
& =0.40
\end{aligned}
$$

\section{Work functions of $\mathrm{MoS}_{2}$}

Work functions for the various compounds were calculated by fitting the high-binding energy cutoff in the UPS spectra for $c e \mathrm{MoS}_{2}$, methyl-MoS 2 , and propyl-MoS 2 synthesized from iodomethane and iodopropane for the latter two. The cutoff value was then used to calculate the work function using the formula $\mathrm{WF}=E(\mathrm{He} \mathrm{I})-\mathrm{BE}$ (cutoff) $=21.2 \mathrm{eV}-\mathrm{BE}$ (cutoff). Values reported in Figure $\mathrm{S} 8$ are the mean of 3 samples \pm standard deviation.

\section{Effective reduction potential for one-electron reductants}

The standard potential for the one-electron redox couple corresponding to each of the reductants used in our experiments was obtained from literature using values obtained in acetonitrile but shifted by $+30 \mathrm{mV}$ based on the difference in formal potentials for the ferrocenium/ ferrocene redox couple in acetonitrile vs DMF. ${ }^{2-3}$ This conversion affects only the comparison between the $\mathrm{MoS}_{2}$ work function and the reductant on the absolute energy scale, for which we used $E_{\mathrm{vac}}=-4.44$ $\mathrm{V}$ vs $\mathrm{SHE}, E\left(\mathrm{Fc}^{+/ 0}\right)=0.544 \mathrm{~V}$ vs $\mathrm{Ag} / \mathrm{AgCl} / \mathrm{KCl}\left(\right.$ sat'd $\left.^{\prime}\right){ }^{3}$ and $E(\mathrm{Ag} / \mathrm{AgCl} / \mathrm{KCl}($ sat'd $))=0.20 \mathrm{~V}$ vs

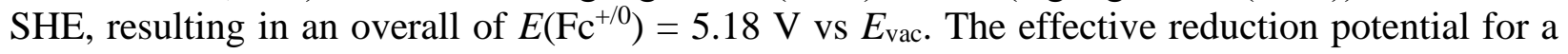
solution where only the reductant was added was estimated from the Nernst equation assuming a 50:1 ratio of reductant to oxidant (i.e. $98 \%$ purity), resulting in a correction of $-0.1 \mathrm{~V}$ to obtain the effective potentials in Figure 4 and Table S2.

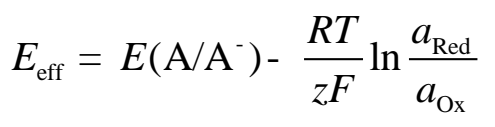




\section{Figures}

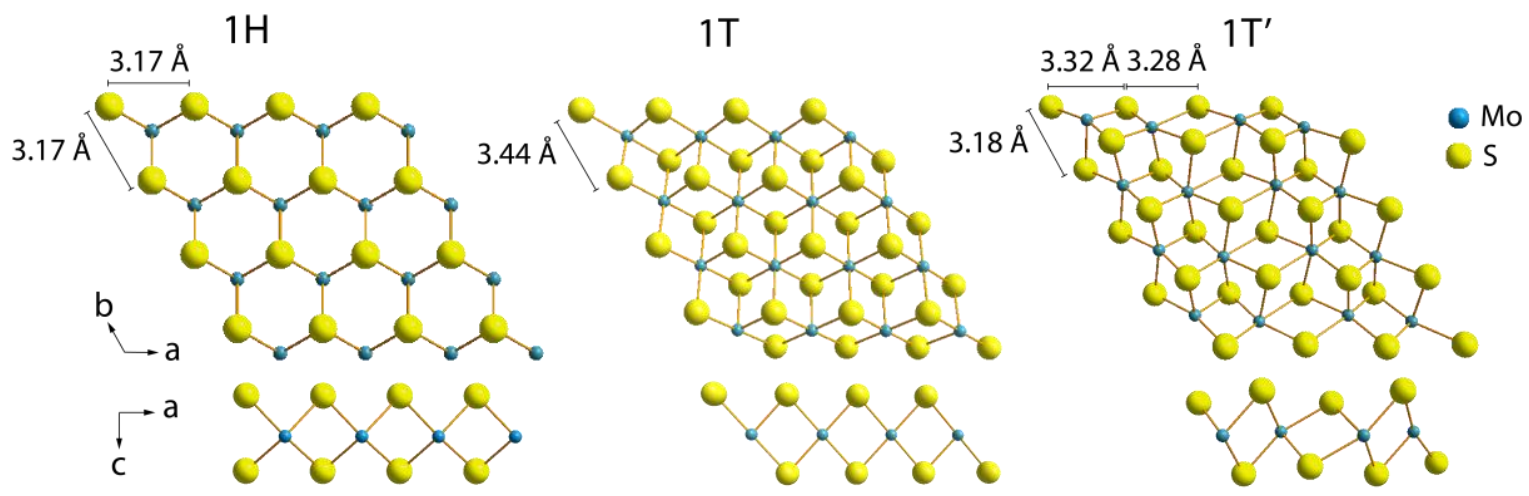

Figure S1. Top-down and side views of $2 \mathrm{H}-$, , $1 \mathrm{~T}-$, and $1 \mathrm{~T}^{\prime}-\mathrm{MoS}_{2}$. Structures of the $2 \mathrm{H}$ and $1 \mathrm{~T}^{\prime}$ phases were generated from crystallographic data, ${ }^{4-5}$ and dimensions for the $1 \mathrm{~T}$ phase obtained from theoretical modeling. ${ }^{6}$

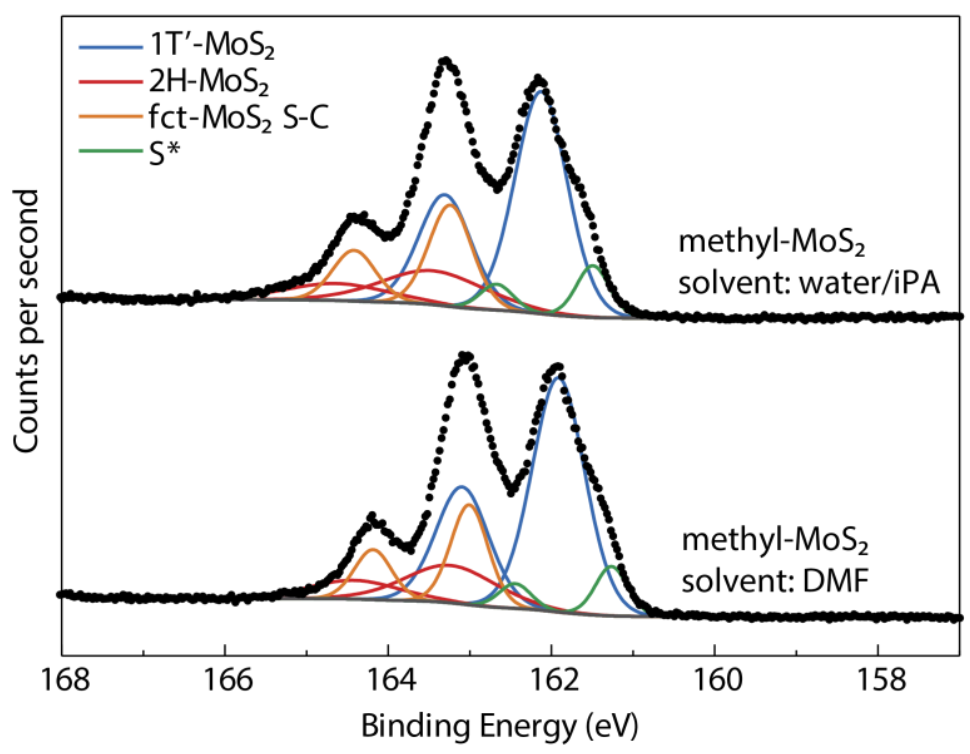

Figure S2. High-resolution XPS of the S $2 p$ region for methyl-MoS $\mathrm{M}_{2}$ synthesized from iodomethane either in a 2:1 water/isopropanol solvent (top) or in $N, N$-dimethylformamide (DMF) (bottom). No substantial differences were observed between the two spectra. 


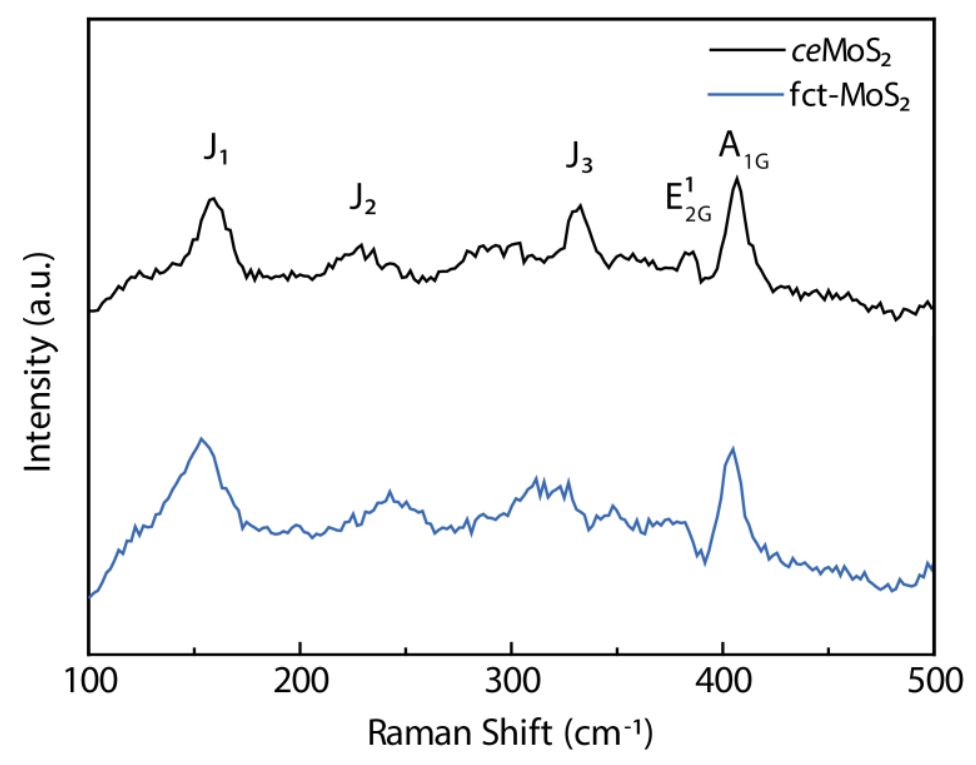

Figure S3. Raman spectra for chemically exfoliated $\mathrm{MoS}_{2}$ before (top black) and after (bottom blue) functionalization. Characteristic peaks at $\sim 159 \mathrm{~cm}^{-1}, 229 \mathrm{~cm}^{-1}$, and $329 \mathrm{~cm}^{-1}$ for the $J_{1}, J_{2}$, and $J_{3}$ vibrational modes are consistent with previously reported values for $c e \mathrm{MoS}_{2}$ and demonstrate that the phase is largely unchanged before and after functionalization. ${ }^{7}$

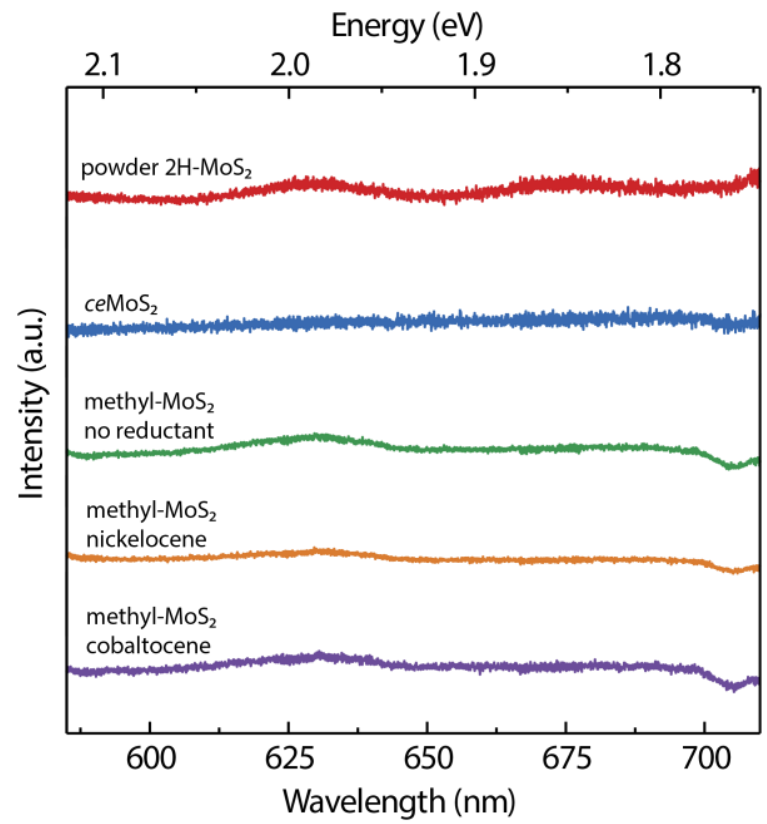

Figure S4. Emission spectra showing photoluminescence region of powder $2 \mathrm{H}-\mathrm{MoS}_{2}$ starting material, chemically exfoliated $\mathrm{MoS}_{2}$, and functionalized $\mathrm{MoS}_{2}$ for methyl-MoS $\mathrm{S}_{2}$ synthesized without reductant, with nickelocene, and with cobaltocene. Excitation was from a $532 \mathrm{~nm}$ diode pumped solid-state laser. Weak to no photoluminescence is observed before or after functionalization in the region where photoluminescence from mono/few-layer $\mathrm{MoS}_{2}$ has typically been observed, ${ }^{8}$ due to the defect-rich nature of the powdered $2 \mathrm{H}-\mathrm{MoS}_{2}$ starting material. 

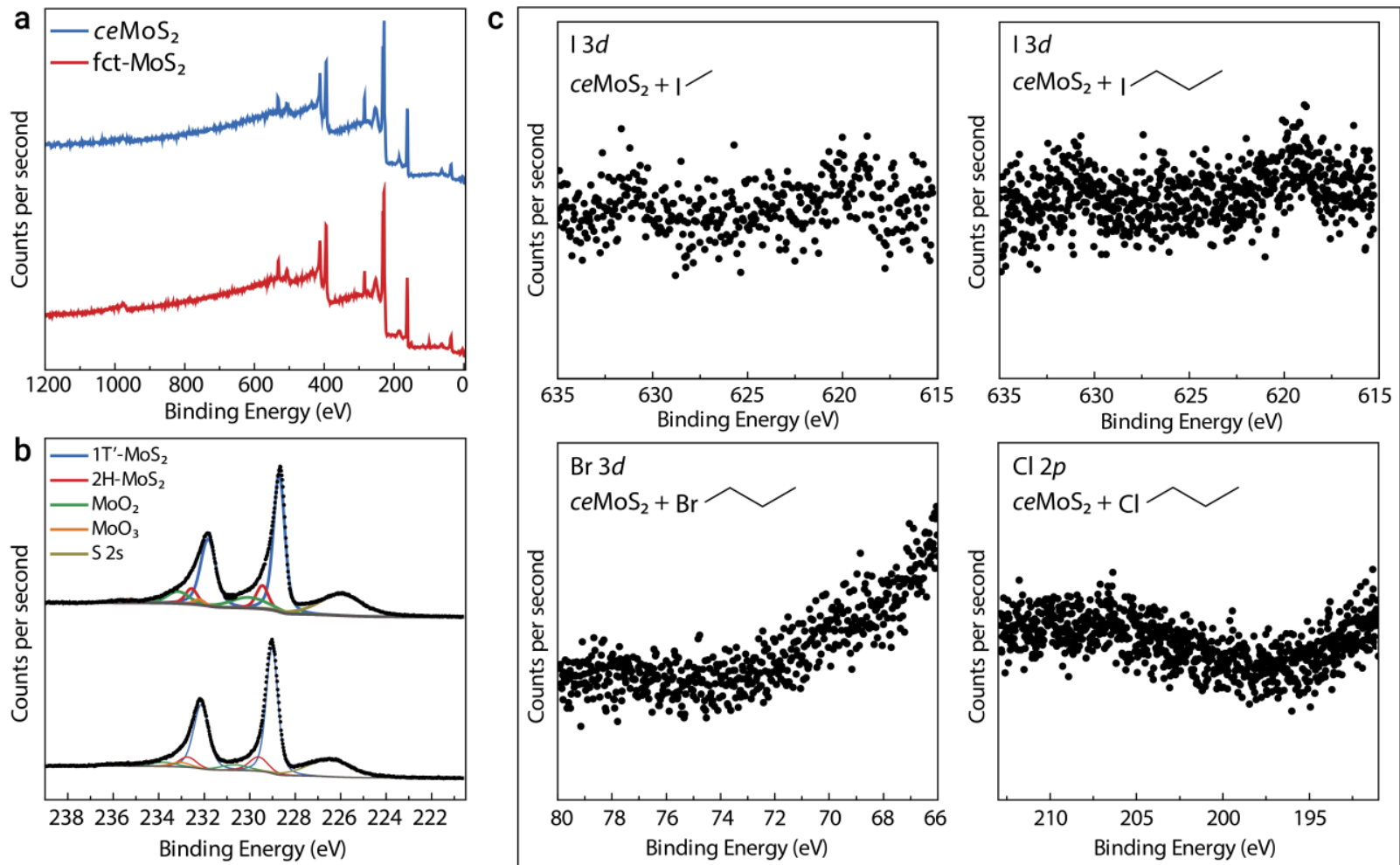

Figure S5. (a) XPS survey and (b) high-resolution XPS of Mo $3 d$ region for $c e \mathrm{MoS}_{2}$ and for functionalized $\mathrm{MoS}_{2}$. No substantial differences were observed between the two spectra. (c) Highresolution XPS data for the halide region of the functionalization molecule (I $3 d, \mathrm{Br} 3 d$, or $\mathrm{Cl} 2 p$ ). 


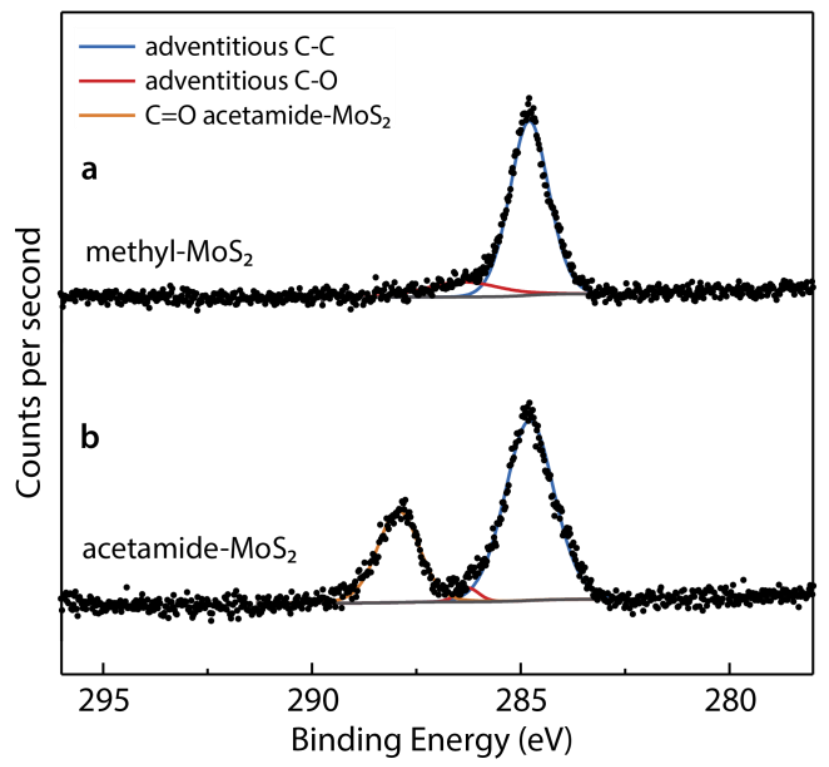

Figure S6. High-resolution XPS of C $1 s$ region for (a) functionalized methyl-MoS $\mathrm{S}_{2}$ and (b) functionalized acetamide- $\mathrm{MoS}_{2}$. Carbon from the carbonyl group is visible in the latter case but not in the former due to overlap with the adventitious carbon peak. The latter result is consistent with previously reported XPS spectra for acetamide- $\mathrm{MoS}_{2}{ }^{7}$

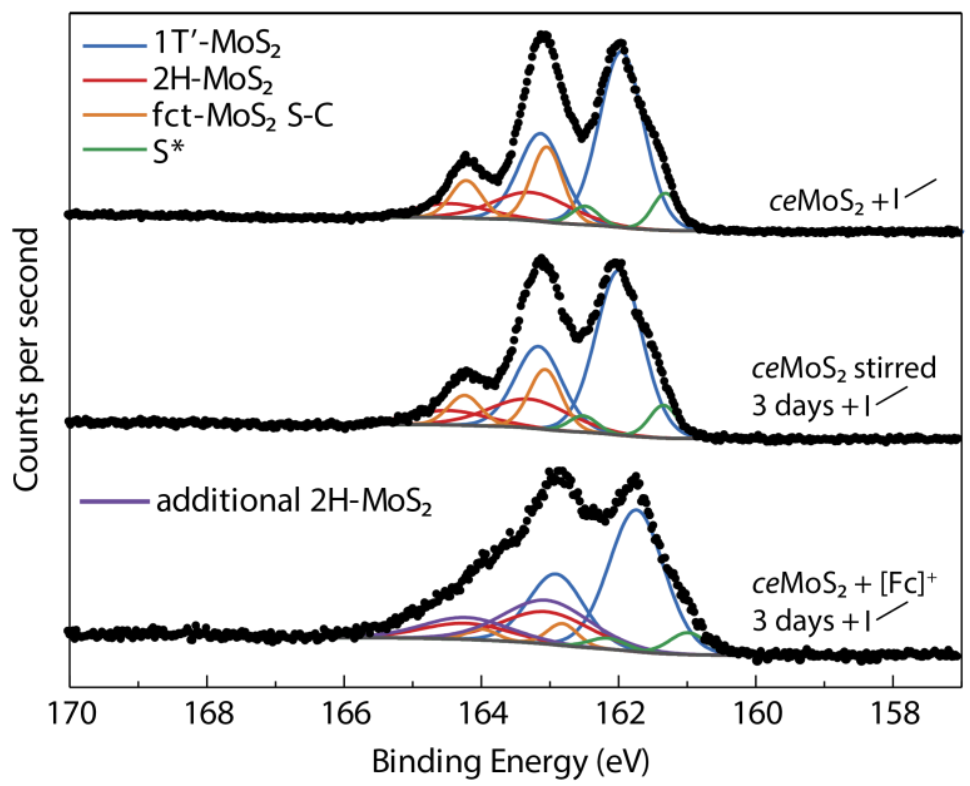

Figure S7. High-resolution XPS of S $2 p$ region for (a) $c e \mathrm{MoS}_{2}$ functionalized with iodomethane following the functionalization procedure from the experimental section, (b) $c e \mathrm{MoS}_{2}$ functionalized with iodomethane after stirring for 3 days under ambient conditions, and (c) $c e \mathrm{MoS}_{2}$ stirred for 3 days with ferrocenium tetrafluoroborate $\left(\left[\mathrm{Fe}\left(\mathrm{C}_{5} \mathrm{H}_{5}\right)_{2}\right] \mathrm{BF}_{4}\right)$ then functionalized with iodomethane. No substantial differences were observed between the first and second conditions. Spectra of samples functionalized after oxidation of $c e \mathrm{MoS}_{2}$ with ferrocenium (c) exhibited S $2 p$ regions with a decreased amount of functionalization and increased amount of $2 \mathrm{H}-\mathrm{MoS}_{2}$ relative to spectra (a) or (b). 

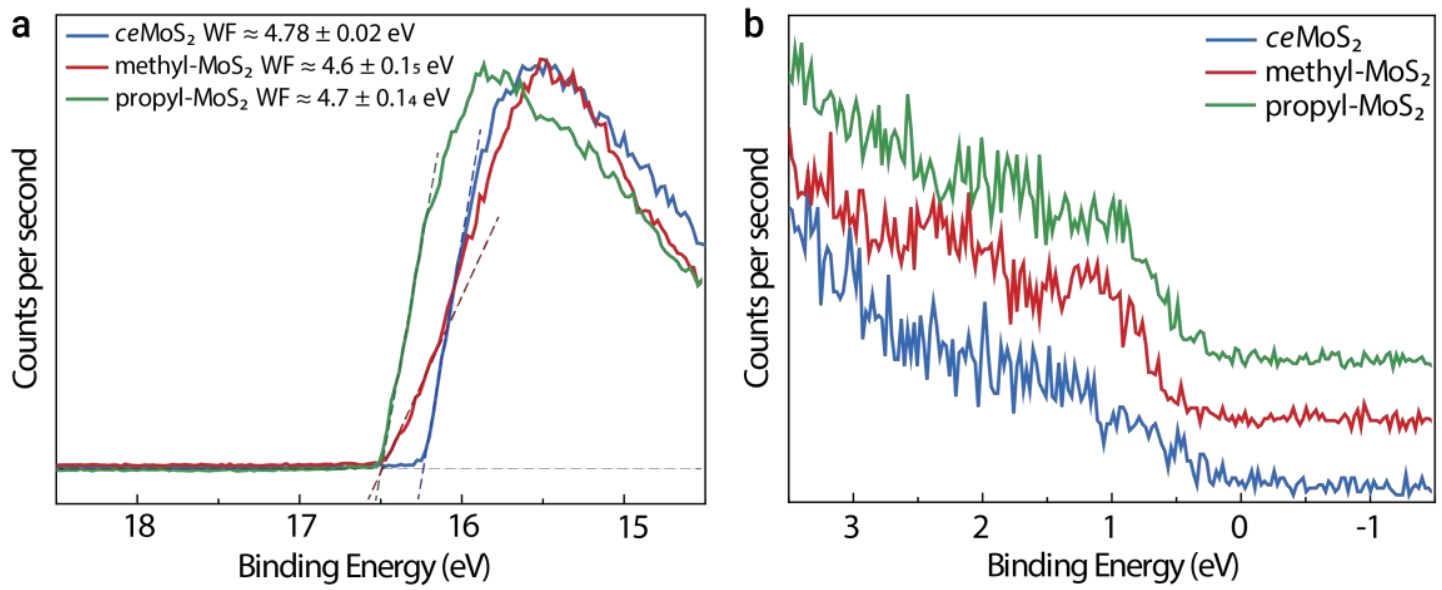

Figure S8. Ultraviolet photoelectron spectra (UPS) for exfoliated $\mathrm{MoS}_{2}$, methyl-MoS $\mathrm{M}_{2}$, and propyl-MoS $\mathrm{M}_{2}$ synthesized using iodomethane and iodopropane, respectively. (a) The high-binding-energy cut-off used to obtain the work function for these powders. Displayed work function and error range is the average of three samples and the standard deviation. (b) Valence-band regime for the same samples, showing a $\leq$ $0.3 \mathrm{eV}$ band gap, consistent with theoretical calculations. ${ }^{9-10}$

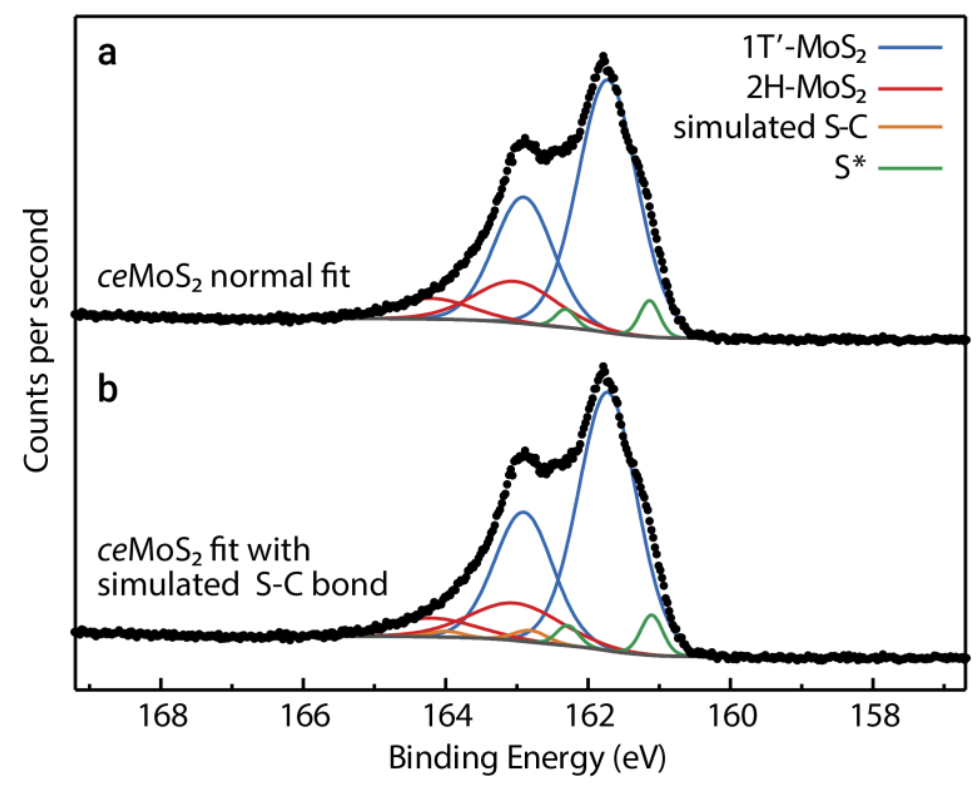

Figure S9. High-resolution XPS of S $2 p$ for exfoliated $\mathrm{MoS}_{2}$ with two types of peak fitting. (a) Three sets of S $2 p$ doublet peaks: one doublet for $1 \mathrm{~T}^{\prime}-\mathrm{MoS}_{2}$, one for $2 \mathrm{H}-\mathrm{MoS}_{2}$, and one for sulfur defects. This peak fitting was used to determine the percentage of sulfur in the $2 \mathrm{H}$ phase in $c e \mathrm{MoS}_{2}$. The percentage of $2 \mathrm{H}-\mathrm{MoS}_{2}$ for all functionalized samples was constrained based on this fitting. (b) Four sets of $\mathrm{S} 2 p$ peaks, the fourth (simulated S-C) constrained to have the same binding energy relative to the $1 \mathrm{~T}^{\prime}-\mathrm{MoS}_{2}$ peak as the functionalized S-C peak observed in fct- $\mathrm{MoS}_{2}$ samples. This peak fitting was used to determine the error associated with peak fitting when using peak areas for coverage quantification (leftmost bar in Figure 4 of main text). 


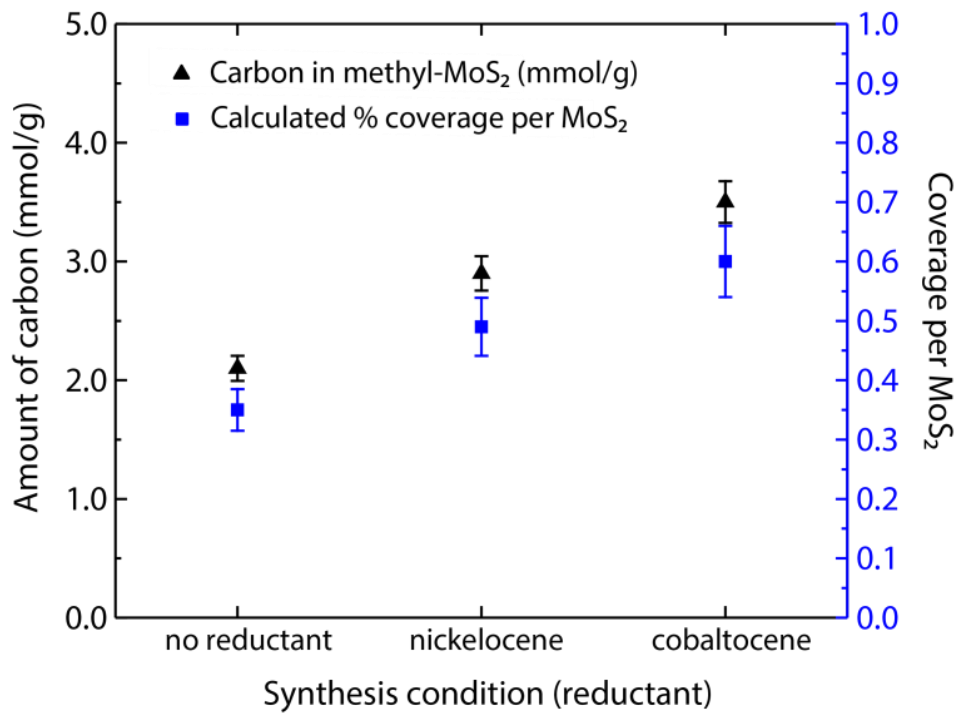

Figure S10. ${ }^{13} \mathrm{C}$ MAS NMR of ${ }^{13} \mathrm{C}$-methyl-MoS 2 functionalized under three conditions: without reductant, with nickelocene, and with cobaltocene. This technique is semi-quantitative and shows the trend of increasing coverage. Error bars indicate $\pm 10 \%$ based on $5 \%$ error of external standard and conservative estimate of $5 \%$ error from peak fitting.
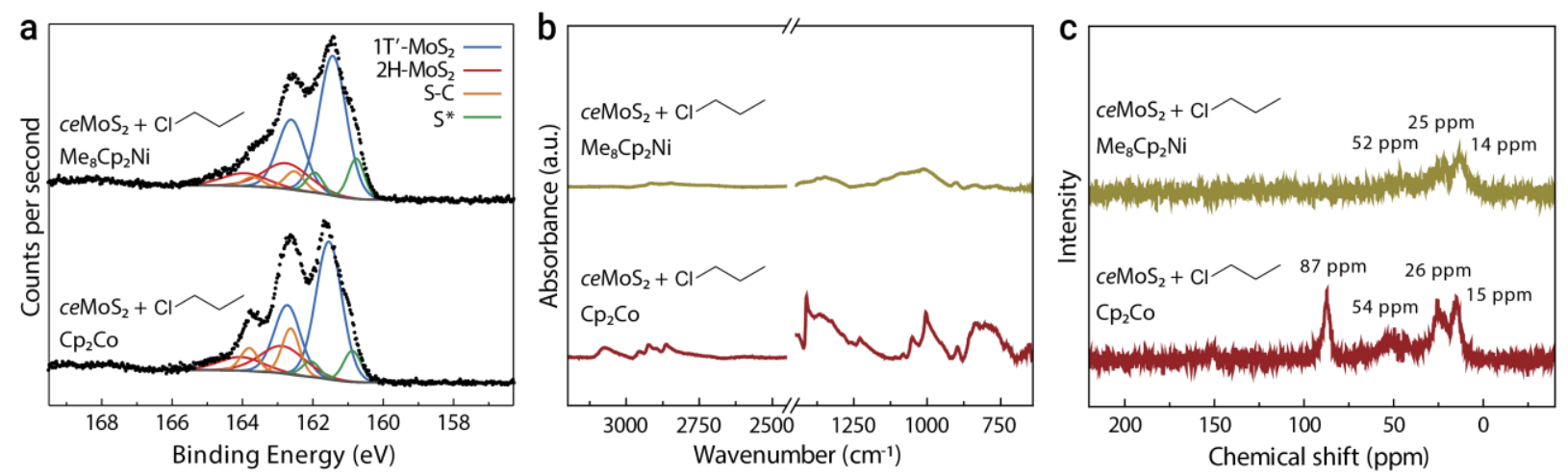

Figure S11. (a) High-resolution XPS of S $2 p$ region, (b) ATR-FTIR, and (c) ${ }^{13}$ C CPMAS NMR data for the functionalization of $c e \mathrm{MoS}_{2}$ with chloropropane in the presence of either octamethylnickelocene $\left(\mathrm{Me}_{8} \mathrm{Cp}_{2} \mathrm{Ni}\right)$ or cobaltocene $\left(\mathrm{Cp}_{2} \mathrm{Co}\right)$. 


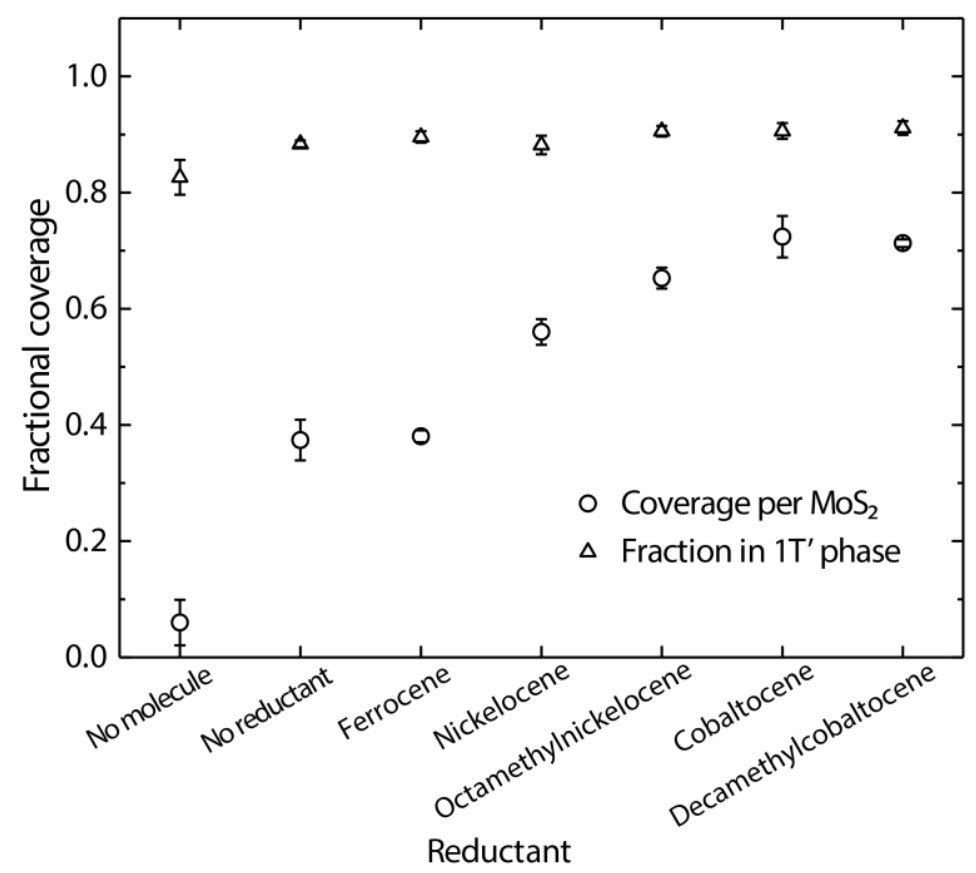

Figure S12. Methyl coverage for methyl-MoS 2 synthesized using iodomethane as a function of the reductant used, and the fraction of $\mathrm{MoS}_{2}$ in the $1 \mathrm{~T}^{\prime}$ phase based on the S $2 p$ XPS peaks. Coverage data are the same as in Figure 4, except with the addition of decamethylcobaltocene $\left(\mathrm{Me}_{10} \mathrm{Cp}_{2} \mathrm{Co}\right)$. Although the latter has a more negative reduction potential than cobaltocene, these results indicate that the coverage does not continue to increase as the reduction potential becomes increasingly negative relative to a limiting value. In addition, the fraction of sulfur in the $1 \mathrm{~T}^{\prime}$ phase is consistently $\sim 90 \%$ in all cases, suggesting that the variation in methyl coverage is not primarily due to the fraction of $1 \mathrm{~T}^{\prime}$ phase. Error bars indicate the standard deviation of at least three samples. 


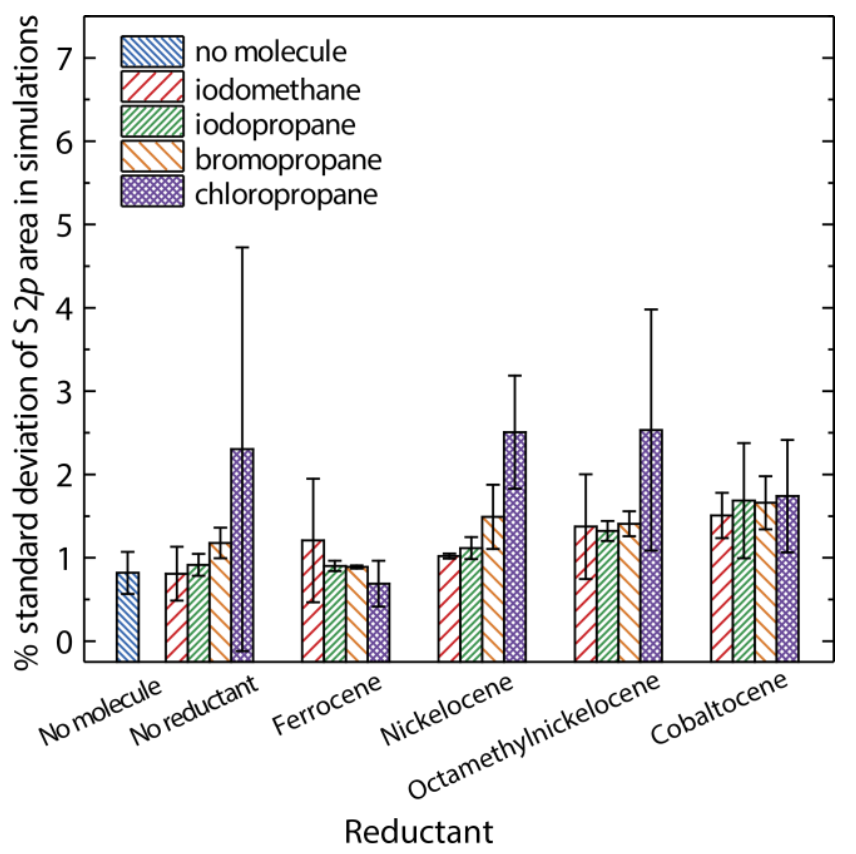

Figure S13. Standard deviations in percentage (\%std) of the functionalized S $2 p$ peak areas fitted during Monte Carlo simulations $(n=400)$ for the data presented in Figure 4. Error bars are the standard deviations for the \%std. Note that the mean for the \%std are below $2 \%$ except for conditions involving chloropropane and minimal functionalization, where a larger \% error is expected. 


\section{Tables}

Table S1: List of all vibrational frequencies calculated using density functional theory (B3LYP with basis set 6-311G**) for methanethiol and propanethiol, assigned to experimental peaks from methyl-MoS $\mathrm{M}_{2}$ and propyl-MoS 2 (synthesized using iodomethane and 1-iodopropane, respectively). A description is provided for characteristic (experimental) or high-intensity (theoretical) peaks. Calculated frequencies that contained substantial contribution from the $\mathrm{S}-\mathrm{H}$ bond were not used to assign peaks due the absence of this bond in experimental samples.

\begin{tabular}{|c|c|c|c|c|}
\hline & $\begin{array}{l}\text { DFT Vibrational } \\
\text { Frequency }\left(\mathrm{cm}^{-1}\right)\end{array}$ & $\begin{array}{l}\text { DFT } \\
\text { Intensity } \\
(\mathbf{k m} / \mathbf{m o l})\end{array}$ & $\begin{array}{l}\text { Experimental Peak } \\
\text { Frequency from } \mathrm{MoS}_{2}-\mathrm{CH}_{3} \\
\text { and } \mathrm{MoS}_{2}-\left(\mathrm{CH}_{2}\right)_{2} \mathrm{CH}_{3}\left(\mathrm{~cm}^{-1}\right)\end{array}$ & Description \\
\hline \multirow[t]{12}{*}{ Methanethiol } & 24500 & 16.1 & -- & -- \\
\hline & 688.57 & 2.56 & 694 & C-S stretch \\
\hline & 800.70 & 1.044 & --- & -- \\
\hline & 978.82 & 11.6 & 946 & C-H in-plane rock \\
\hline & 1099.55 & 20.8 & --- & $\mathrm{CH}_{3} / \mathrm{S}-\mathrm{H}$ rock \\
\hline & 1369.87 & 10.4 & 1289 & $\mathrm{C}-\mathrm{H}$ symmetrical wag $\left(\mathrm{CH}_{3}\right)$ \\
\hline & 1475.65 & 5.49 & --- & -- \\
\hline & 1486.35 & 8.65 & -- & -- \\
\hline & 2659.60 & 8.76 & 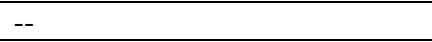 & S-H stretch \\
\hline & 3047.06 & 28.6 & 2905 & C-H symmetrical stretch $\left(\mathrm{CH}_{3}\right)$ \\
\hline & 3130.39 & 10.8 & 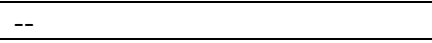 & -- \\
\hline & 3135.79 & 8.24 & -- & -- \\
\hline \multirow{30}{*}{ Propanethiol } & 108.54 & 4.44 & -- & -- \\
\hline & 174.96 & 17.7 & -- & -- \\
\hline & 231.84 & 2.22 & -- & -- \\
\hline & 249.30 & 0.0700 & -- & -- \\
\hline & 355.97 & 0.528 & --- & -- \\
\hline & 721.89 & 3.44 & 727 & C-S stretch \\
\hline & 768.36 & 4.94 & 793 & $\mathrm{C}-\mathrm{H}$ in-plane rock $\left(\mathrm{CH}_{3} / \mathrm{CH}_{2}\right)$ \\
\hline & 847.50 & 3.66 & -- & S-H bend \\
\hline & 882.49 & 0.334 & -- & -- \\
\hline & 944.17 & 1.581 & 898 & $\begin{array}{l}\text { C-H out-of-plane wag } \\
\left(\mathrm{CH}_{3} / \mathrm{CH}_{2}\right)\end{array}$ \\
\hline & 1043.98 & 0.0873 & -- & -- \\
\hline & 1074.90 & 5.08 & 1054 & $\mathrm{C}-\mathrm{H}$ twist $\left(\mathrm{CH}_{2} / \mathrm{CH}_{3}\right)$ \\
\hline & 1135.96 & 5.40 & 1082 & C-C-C symmetric stretch \\
\hline & 1250.33 & 0.960 & 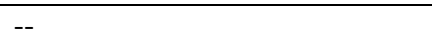 & -- \\
\hline & 1283.66 & 37.2 & 1257 & $\mathrm{C}-\mathrm{H}$ wag $\left(\mathrm{CH}_{2}\right)$ \\
\hline & 1334.70 & 3.45 & --- & -- \\
\hline & 1383.15 & 4.97 & -- & -- \\
\hline & 1418.79 & 2.79 & -- & -- \\
\hline & 1491.30 & 2.52 & -- & -- \\
\hline & 1499.35 & 1.56 & -- & -- \\
\hline & 1502.66 & 8.80 & -- & -- \\
\hline & 1511.67 & 5.58 & -- & -- \\
\hline & 2662.37 & 9.01 & -- & S-H stretch \\
\hline & 3020.12 & 22.4 & 2862 & $\mathrm{C}-\mathrm{H}$ symmetric stretch $\left(\mathrm{CH}_{3}\right)$ \\
\hline & 3023.59 & 11.0 & -- & -- \\
\hline & 3043.41 & 40.4 & 2924 & $\mathrm{C}-\mathrm{H}$ symmetric stretch $\left(\mathrm{CH}_{2}\right)$ \\
\hline & 3050.68 & 1.07 & -- & -- \\
\hline & 3081.84 & 17.6 & -- & -- \\
\hline & 3091.20 & 36.7 & \multirow[t]{2}{*}{2955} & $\mathrm{C}-\mathrm{H}$ asymmetric stretch $\left(\mathrm{CH}_{3}\right)$ \\
\hline & 3098.89 & 50.4 & & $\begin{array}{l}\text { C- }-\mathrm{H}_{\text {asymmetric stretch }} \\
\left(\mathrm{CH}_{2} / \mathrm{CH}_{3}\right)\end{array}$ \\
\hline
\end{tabular}


Table S2: The standard reduction potential for each redox couple, the effective reduction potential assuming $50: 1$ ratio of reductant to oxidant, and the potential on an absolute energy scale using $E\left(\mathrm{Fc}^{+/ 0}\right)=0.74 \mathrm{~V}$ vs SHE (standard hydrogen electrode) and $E_{\mathrm{vac}}=-4.44 \mathrm{~V}$ vs SHE (see supplementary section III.4).

\begin{tabular}{|c|c|c|c|}
\hline Reductant (A-) & $\begin{array}{l}\text { Standard Reduction Potential } \\
\text { for }\left(\mathbf{A} / \mathbf{A}^{-}\right) \text {vs } E\left(\mathbf{F c}^{+/ 0}\right)(\mathrm{V})\end{array}$ & $\begin{array}{l}\text { Effective Reduction Potential, } 50: 1 \\
\text { reductant to oxidant vs } E\left(\mathrm{Fc}^{+/ 0}\right)(\mathrm{V})\end{array}$ & $\begin{array}{l}\text { Potential vs } \\
\mathbf{E}_{\text {vac }}(\mathrm{V})\end{array}$ \\
\hline ferrocene, $\mathrm{Cp}_{2} \mathrm{Fe}$ & 0 & -0.10 & 5.08 \\
\hline nickelocene, $\mathrm{Cp}_{2} \mathrm{Ni}$ & -0.42 & -0.52 & 4.66 \\
\hline $\begin{array}{l}\text { octamethylnickelocene, } \\
\mathrm{Me}_{8} \mathrm{Cp}_{2} \mathrm{Ni}\end{array}$ & -0.95 & -1.05 & 4.13 \\
\hline cobaltocene, $\mathrm{Cp}_{2} \mathrm{Co}$ & -1.16 & -1.26 & 3.92 \\
\hline $\begin{array}{l}\text { decamethylcobaltocene, } \\
\mathrm{Me}_{10} \mathrm{Cp}_{2} \mathrm{Co}\end{array}$ & -1.77 & -1.87 & 3.31 \\
\hline
\end{tabular}




\section{References}

(1) Baltrusaitis, J.; Mendoza-Sanchez, B.; Fernandez, V.; Veenstra, R.; Dukstiene, N.; Roberts, A.; Fairley, N. Generalized molybdenum oxide surface chemical state XPS determination via informed amorphous sample model. Applied Surface Science 2015, 326, 151-161.

(2) Grimm, R. L.; Bierman, M. J.; O’Leary, L. E.; Strandwitz, N. C.; Brunschwig, B. S.; Lewis, N. S. Comparison of the Photoelectrochemical Behavior of H-Terminated and MethylTerminated $\mathrm{Si}(111)$ Surfaces in Contact with a Series of One-Electron, Outer-Sphere Redox Couples in $\mathrm{CH}_{3} \mathrm{CN}$. J. Phys. Chem. C 2012, 116, 23569-23576.

(3) Noviandri, I.; Brown, K. N.; Fleming, D. S.; Gulyas, P. T.; Lay, P. A.; Masters, A. F.; Phillips, L. The Decamethylferrocenium/Decamethylferrocene Redox Couple: A Superior Redox Standard to the Ferrocenium/Ferrocene Redox Couple for Studying Solvent Effects on the Thermodynamics of Electron Transfer. J. Phys. Chem. B 1999, 103, 6713-6722.

(4) Voudouris, P. C.; Melfos, V.; Spry, P. G.; Bindi, L.; Kartal, T.; Arikas, K.; Moritz, R.; Ortelli, M. Rhenium-rich Molybdenite and Rheniite in the Pagoni Rachi $\mathrm{Mo}-\mathrm{Cu}-\mathrm{Te}-\mathrm{Ag}-\mathrm{Au}$ Prospect, Northern Greece: Implications for the Re Geochemistry of Porphyry-style $\mathrm{Cu}-\mathrm{Mo}$ and Mo Mineralization. The Canadian Mineralogist 2009, 47, 1013-1036.

(5) Calandra, M. Chemically exfoliated single-layer $\mathrm{MoS}_{2}$ : Stability, lattice dynamics, and catalytic adsorption from first principles. Phys. Rev. B 2013, 88, 245428.

(6) Qu, Y.; Pan, H.; Kwok, C. T. Hydrogenation-controlled phase transition on two-dimensional transition metal dichalcogenides and their unique physical and catalytic properties. Sci. Rep. 2016, $6,34186$.

(7) Voiry, D.; Goswami, A.; Kappera, R.; Silva, C. d. C. C. e.; Kaplan, D.; Fujita, T.; Chen, M.; Asefa, T.; Chhowalla, M. Covalent functionalization of monolayered transition metal dichalcogenides by phase engineering. Nat. Chem. 2015, 7, 45-49.

(8) Eda, G.; Yamaguchi, H.; Voiry, D.; Fujita, T.; Chen, M.; Chhowalla, M. Photoluminescence from chemically exfoliated $\mathrm{MoS}_{2}$. Nano Lett. 2011, 11, 5111-5116.

(9) Chou, S. S.; Sai, N.; Lu, P.; Coker, E. N.; Liu, S.; Artyushkova, K.; Luk, T. S.; Kaehr, B.; Brinker, C. J. Understanding catalysis in a multiphasic two-dimensional transition metal dichalcogenide. Nat. Commun. 2015, 6, 8311.

(10) Fan, X.-L.; Yang, Y.; Xiao, P.; Lau, W.-M. Site-specific catalytic activity in exfoliated $\mathrm{MoS}_{2}$ single-layer polytypes for hydrogen evolution: basal plane and edges. J. Mater. Chem. A 2014, 2, 20545-20551. 